\title{
Bases moléculaires du mouvement flagellaire
}

\author{
J-L. GatTi, J-L DacheUX
}

URA 1291 INRA-CNRS, Station de Physiologie de la Reproduction des Mammiferes

Domestiques. 37380 Monnaie, France.

\section{RESUME}

Au cours de l'évolution, pour pouvoir se déplacer dans le milieu qui les entoure, les cellules ont développé des structures spécialisées : les cils et les flagelles. Ces structures qui produisent des successions d'ondes symétriques ou asymétriques, contiennent la même "machinerie" interne, l'axonème.

L'axonème est composé généralement de neuf doublets de microtubules externes entourant une paire centrale de microtubules. Le battement résulte d'un phénomène actif, dû à l'attachement temporaire des dynéine-ATPases d'un doublet externe au doublet adjacent. Il se produit alors un glissement entre les doublets qui est transformé en courbure par les résistances de certaines structures axonémale. Les mécanismes moléculaires impliqués dans la génération du mouvement sont les même dans les cils et les flagelles, bien que les voies de contrôle de ces mécanismes puissent être différents.

Cette revue se présente en deux parties : la première est un rappel sur la composition de l'axonème incluant les récentes découvertes aux niveaux ultrastructural et biochimique, la seconde porte sur la mécanique du battement axonémal et présente le rôle de certaines des structures ou des protéines dans la génération du battement.
Mots clés : flagelles, cils, dyneines, battement flagellaire, spermatozoïdes.

\section{INTRODUCTION}

Au cours de l'évolution, pour pouvoir se déplacer dans le milieu qui les entoure, les cellules ont développé des structures spécialisées : les cils et les flagelles. Ces structures de 0,1 à $1 \mathrm{~mm}$ de diamètre et de 10 à plus de $100 \mathrm{~mm}$ de long, produisent un battement qui est une succession d'ondes symétriques ou asymétriques se propageant généralement de la base vers le sommet de ces appendices. Ces cils et ces flagelles contiennent la même "machinerie" interne, un cylindre d'environ $250 \mathrm{~nm}$ de diamètre: l'axonème. Celui-ci présente en général une structure dite " $9+2$ " qui est un assemblage de neuf doublets de microtubules externes entourant une paire centrale de microtubules (Figure 1).

Les mécanismes moléculaires impliqués dans la génération du mouvement sont les mêmes dans les cils et les flagelles, bien que les voies de contrôle de ces mécanismes puissent être différentes. Schématiquement, le mouvement de l'axonème résulte du glissement coordonné entre les doublets de microtubules externes sous l'action des dynéines-ATPases, extensions portées par les bras externes et internes, et de la résistance qu'opposent à ce glissement certaines des autres structures axonémales. 


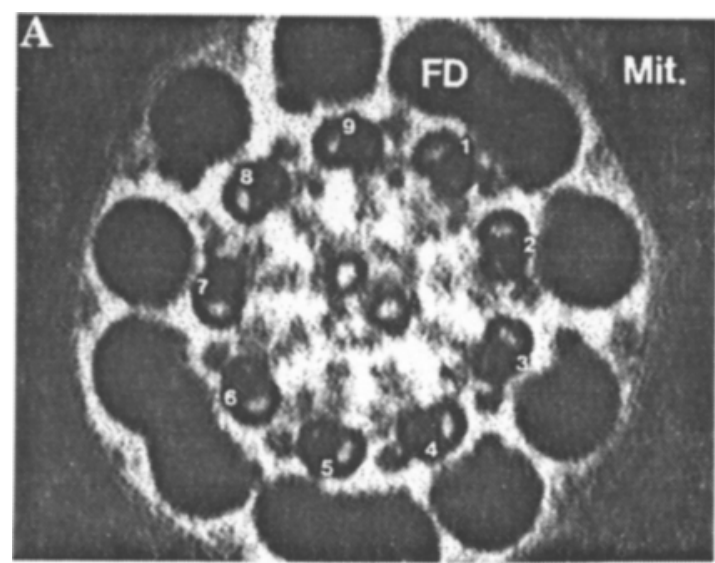

Figure 1 : Structure de l'axonème.

En A : photomicrographie d'une section transversale de flagelle de spermatozoïde de bélier. L'axonème de type 9+2 est entouré par les 9 fibres denses (FD) et l'anneau de mitochondries (Mit.). Les doublets sont numérotés de 1 à 9. Grossissement 240 000.

Actuellement, les principales découvertes sur la structure et le rôle des protéines axonémales dans le battement flagellaire proviennent de l'étude de l'algue unicellulaire biflagellée Chlamydomonas. Chez ce protiste il est possible d'induire des mutants et de les sélectionner sur leurs problèmes de mobilité et/ou de battement flagellaire.

Cette revue se présente en deux parties: une est un rappel sur la composition de l'axonème en incluant les récentes découvertes aux niveaux ultrastructural et biochimique, la seconde porte sur la mécanique du battement axonémal et présente le rôle de certaines des structures ou des protéines dans ce mouvement.

\section{STRUCTURE}

L'axonème est la structure stable des cils et des flagelles restant après l'extraction de la membrane et du cytosol par un détergent. Cette structure minimale est suffisante pour que le battement flagellaire ait lieu. Ceci est montré par la possibilité de réactiver le mouvement de ces axonèmes en présence d'ATP et de $\mathrm{Mg} 2+$ [31].

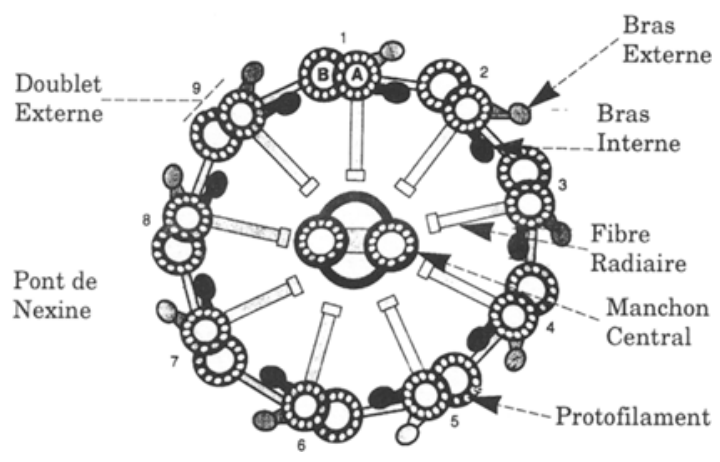

Figure 1 : Structure de l'axonème.

En $B$ : Schéma representant l'axonème. Les doublets externes résultent de la fusion de deux microtubules : le microtubule A porte les bras externe et interne. Le manchon central contient la paire de microtubules centraux entourée de projections. Chaque microtubule est formé par l'assemblage de 13 protofilaments d' $\alpha$ et $\beta$ tubuline (voir texte).

Bien que présentant certaines variations entre les espèces, l'axonème est constitué par un assemblage microtubulaire complexe entouré ou non par des structures dites accessoires.

\section{Les microtubules}

Ce sont de petits tubes de $25 \mathrm{~nm}$ de diamètre dont la paroi est formée par l'association de 13 protofilaments (Figure 1). Chaque protofilament résulte de l'empilement d'hétérodimère composé d' $\alpha$ et de $\beta$ tubuline [61]. Les 9 doublets externes proviennent de la fusion de deux microtubules qui partagent trois protofilaments sur toute leur longueur: ainsi, le microtubule A qui apparaît circulaire possède 13 protofilaments, et le microtubule tronqué $\mathrm{B}$ n'en possède que 10. Dans de nombreuses espèces la lumière d'un des deux tubules est bouchée par la présence de matériel ou de projections $[2,112]$. Les doublets peuvent être numérotés de 1 à 9 , le numéro 1 étant situé dans l'axe passant entre les microtubules du doublet central. Cet axe représente aussi le plan dans lequel se développe le battement flagellaire. 
Dans l'axonème, les microtubules sont extrêmement stables. Cette stabilité semble due à différents facteurs $[34,61,78]$ :

1. à des modifications post-transcriptionelles de la tubuline $\alpha$ (poly-glutamylation, détyrosination),

2. à la présence d'un variant de cette tubuline $\alpha$ (nommée delta 2 -tubuline) qui est amputée des deux acides aminés carboxyterminaux,

3. à la présence dans les microtubules externes et centraux de protéines apparentées aux filaments intermédiaires (tektines). Chez les spermatozoïdes d'oursin, une protéine proche de l'histone $\mathrm{H} 1$ pourrait aussi jouer un rôle dans la stabilité [70].

\section{Les bras externes et internes}

Chacun des microtubules A des neuf doublets externes porte des extensions en forme de crochets, dirigées vers le microtubule $\mathrm{B}$ du doublet adjacent: ce sont les bras externes et internes. Ces bras indiquent le sens horaire quand les coupes d'axonèmes sont vues depuis la base vers le sommet.

Ces bras sont formés par des complexes protéiques à activité ATPasique, les dynéines; ce sont les moteurs moléculaires du battement flagellaire. Ces ATPases sont uniques par leur taille (jusqu'à 1,5 MDa), leur composition (plus de 15 protéines) et peuvent contenir plusieurs chaînes de hauts poids moléculaires possédant un site catalytique actif.

Les bras externes sont distribués le long du microtubule avec une grande régularité bien que l'espacement entre ces bras ne soit pas le même chez toutes les espèces: il y a un bras tous les $24 \mathrm{~nm}$ chez Chlamydomonas. Les bras internes sont distribués de façon plus complexe : ils sont groupés par trois (nommés I1, I2, et I3) et chaque groupe se répète tous les $96 \mathrm{~nm}$ le long du microtubule (Figure 2). La distance entre I1 et $\mathrm{I} 2$ est de $32 \mathrm{~nm}$, celle entre

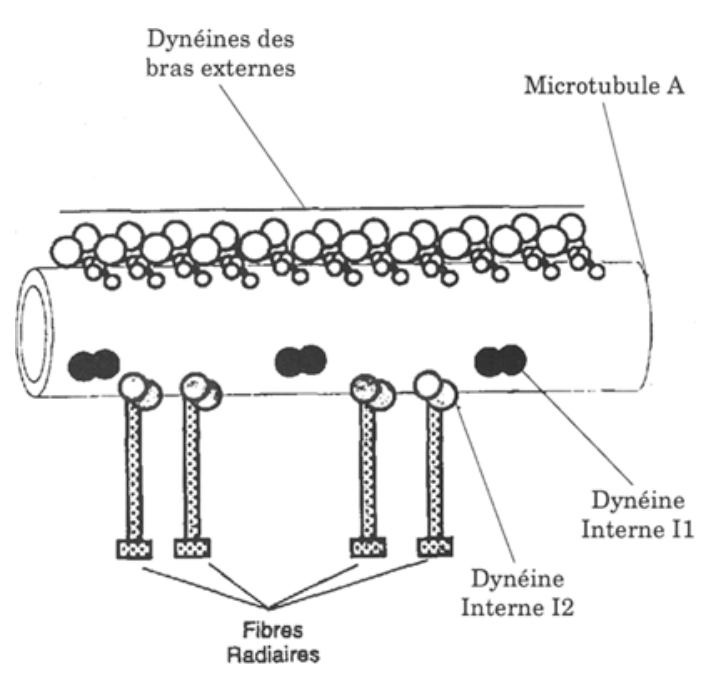

Figure 2 : Microtubule A vue de face. Schéma de la position des dynéines des bras externes et internes le long du microtubule A vue depuis le microtubule $B$ adjacent chez Chlamydomonas (voir texte). D'après l'analyse en microscopie des réfèrences 60,71 .

I 2 et I3 est de $24 \mathrm{~nm}$. Il y a $40 \mathrm{~nm}$ entre le bras $I 3$ et le bras $I 1$ suivant $[81,83]$. Chez Chlamydomonas, les bras internes ne sont pas alignés en un seul rang, mais forment deux rangées longitudinales avec le bras I2 plus intérieur que I1 (Figure 2), mais cette disposition pourrait être spécifique $[60,71]$.

Chez la plupart des espèces, les bras externes et internes forment une liaison stable entre deux des doublets externes : entre les doublets 9-1 chez Chlamydomonas, 5-6 chez l'oursin et chez le rat $[91,56,112]$.

\section{Les liaisons entre les doublets externes}

Chacun des doublets externes est connecté à son voisin par une projection, le pont de nexine. Cette liaison de $25 \mathrm{~nm}$ relie le microtubule $\mathrm{A}$ au dos du microtubule $\mathrm{B}$ lui faisant face. Ces liens de nexine se répètent tout le long du microtubule avec une périodicité de 96 nm [113]. Chez Chlamydomonas des liens supplémentaires entre les microtubules $\mathrm{A}$ de doublets adjacents sont présents dans la partie proximale de l'axo- 
nème : ce sont les liens périphériques. Ces liens ont environ $40 \mathrm{~nm}$ de long et relient la base des fibres radiaires des doublets périphériques $[6,113]$.

\section{Les fibres radiaires}

De chaque microtubule A des doublets externes part une extension dirigée vers les microtubules centraux: ce sont les rayons ou fibres radiaires. Ces fibres se terminent par un renflement du coté central [20]. Le long du doublet externe ces fibres radiaires sont groupées par deux ou trois suivant les espèces (nommées $\mathrm{S} 1, \mathrm{~S} 2, \mathrm{~S} 3$ ) avec une périodicité de $96 \mathrm{~nm}$ (Figure 2). La fibre S2 est proche de l'emplacement du lien de nexine.

\section{Le manchon central}

Il est constitué par un doublet central de deux microtubules de 13 protofilaments chacun, joints entre eux par des ponts espacés de $16 \mathrm{~nm}$ [113]. Ces microtubules portent chacun des projections qui les enrobent, cet ensemble forme le manchon central. La forme et la distribution des projections le long de ces microtubules varient suivant les espèces.

\section{Structures accessoires}

Chez les spermatozoïdes de nombreuses espèces, et en particulier chez les mammifères, autour de l'axonème $9+2$ se sont développés des structures accessoires [4,7]. Chez les mammifères, ces structures changent suivant le niveau flagellaire. De la jonction tête-flagelle jusqu'à la fin de la pièce principale, à chacun des doublets périphériques est associée une fibre dense (axonème $9+9+2$ ). Ces neuf fibres ont des tailles, des formes et des longueurs différentes [94]. Autour de ces fibres, il existe aussi une gaine fibreuse formée par deux colonnes longitudinales reliées entre elles par des cotes. Dans la partie distale de la pièce principale, les colonnes se substituent aux fibres denses des doublets 3 et 8 . Il semble qu'il y ait une continuité fonctionnelle et structurale suivant l'axe 3-8 entre les colonnes, les fibres denses, les doublets externes et le doublet central [56].

\section{Le corps basal}

A l'extrémité proximale, de l'axonème se trouve le corps basal qui est constitué de neuf triplets de microtubules. Ce corps basal sert de racine lors de l'élaboration de l'axonème par la cellule et certainement à restreindre le mouvement entre les doublets de microtubules à ce niveau.

Il y a une relation étroite entre ce corps basal et les centrioles, et ceci est flagrant pour les spermatozoïdes. En effet, lors de la spermatogénèse, le centriole distal sert de point de départ pour le développement du flagelle. Chez les mammifères, ce centriole devient partie intégrante de l'axonème et disparaît quasiment lorsque la jonction tête flagelle est terminée [7]. Des anomalies génétiques au niveau de ce centriole ou de sa position lors de la spermatogénèse entraînent des malformations flagellaires et des stérilités [1].

Dans les cellules ciliées et chez les spermatozoïdes d'invertébrés, le corps basal s'entoure de structures fibreuses à l'architecture complexe qui servent à ancrer l'axonème [112].

\section{COMPOSITION BIOCHIMIQUE}

Lorsque des axonèmes purifiés sont analysés par gel d'électrophorèse bidimensionel, près de 200 polypeptides différents peuvent être observés [58]. Par la même technique, l'analyse d'axonèmes dont certaines structures ont été extraites, ou sont absentes à la suite d'une mutation, montre que la plupart de celles-ci sont des complexes formés de plusieurs protéines.

\section{Les microtubules}

Les tubulines $\alpha$ et $\beta$ qui forment les microtubules représentent environ $70 \%$ des protéines totales de l'axonème. Ces protéines, qui ont une masse apparente d'environ 50 $\mathrm{kDa}$, sont codées par deux gènes différents 
[16]. Elles possèdent respectivement 436 et 430 acides aminés dont la séquence est hautement conservée à l'exception de la partie $\mathrm{C}$ terminale. Ces protéines ont un site catalytique qui reconnaît le GTP $[16,61]$. L'hydrolyse du GTP intervient lors de la polymérisation des microtubules.

\section{Les complexes de dynéines}

\section{a) Les dynéines des bras externes.}

Chez différentes espèces, les axonèmes peuvent être isolés après une déflagellation chimique ou mécanique en présence de détergent. A partir de ces axonèmes les bras externes ont été extraits par un traitement à haute force ionique $[8,26,111]$. Cette méthode permet en général la solubilisation de plus de $50 \%$ de l'activité ATPasique de l'axonème et l'extraction de $90 \%$ des bras externes. La solution d'extraction est ensuite fractionnée par ultracentrifugation sur gradient de saccharose. Suivant l'espèce, soit une particule avec un coefficient de sédimentation de $\sim 20 \mathrm{~S}$, soit plusieurs particules à $\sim 20 \mathrm{~S}$ et $\sim 11 \mathrm{~S}$ sont obtenues. Chacune de ces particules possède une activité ATPasique. Il est possible de reconstituer le bras externe en mélangeant ces particules purifiées et les axonèmes extraits. Il semble cependant qu'une protéine de $70 \mathrm{kDa}$ extraite des microtubules par la haute force ionique soit nécessaire à une reconstruction complète [100].

Les particules migrant à 20S apparaissent en microscopie électronique comme un bouquet de 30 à $35 \mathrm{~nm}$ constitué, suivant l'espèce, de deux ou de trois domaines globulaires ou ovoïdes (têtes) de 10 à $15 \mathrm{~nm}$ portés chacun par une tige qui émerge d'un pied commun [33] (Figure 3). La masse de ces particules a pu être estimée par microscopie de transmission (STEM) : une particule à trois têtes fait $1.9 \mathrm{MDa}$ et une à deux têtes 1,2-1,3 MDa [41,59]. Chaque domaine globulaire représente environ $300 \mathrm{kDa}$. Les particules migrant à $11 \mathrm{~S}$ sont constituées d'une seule tête et d'une tige. Leur masse par STEM est d'environ $500 \mathrm{kDa}[41,59]$.

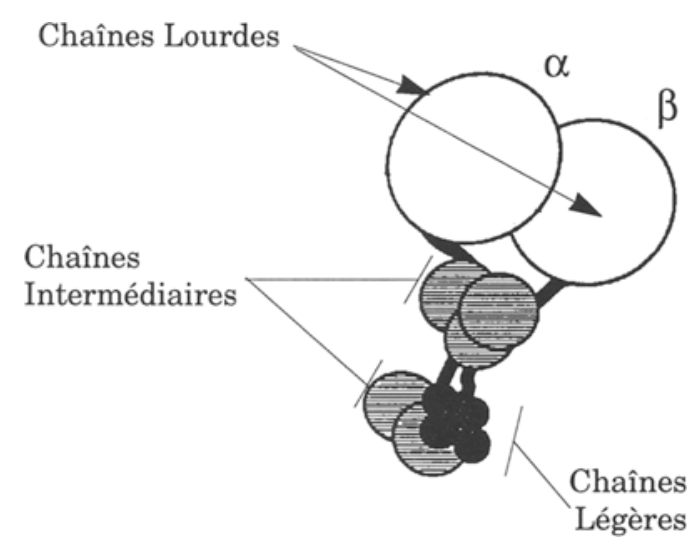

Figure 3 : Dynéine du bras externe. Représentation schématique de la dynéine de bras externe extraite des flagelles de spermatozoïdes de truite. La position des différentes chaîne est interprétée d'après les complexes obtenus lors de la dissociation à faible force ionique [26, 50$]$.

L'analyse de ces particules, par gel d'électrophorèse, montre qu'elles sont constituées d'une dizaine de protéines différentes variant entre 5 et $600 \mathrm{kDa}[111,114]$ (Tableau 1). Trois catégories de protéines ont été définies : les chaînes de hauts poids moléculaires (chaînes lourdes) au dessus de $400 \mathrm{kDa}$, les chaînes intermédiaires entre 50 et $150 \mathrm{kDa}$, et les chaînes légères en dessous de $50 \mathrm{kDa}$. Le nombre de chaînes lourdes est de deux ou de trois pour les particules de $20 \mathrm{~S}$ (nommées par des lettres grecques depuis la plus lourde) et de 1 pour les particules de $11 \mathrm{~S}$. Ce nombre est le même que celui des domaines globulaires observés par microscopie.

Les résultats obtenus chez les différentes espèces semblent indiquer que le nombre de chaînes lourdes contenues dans les dynéines de bras externes est de deux chez les métazoaires alors qu'il est de trois chez les protozoaires (Tableau 1). Cependant cette différence pourrait aussi être celle entre les dynéines ciliaires et flagellaires. Il n'y a pas de règle apparente dans la distribution des chaînes légères et intermé- 


\section{Nombre de chaînes}

\begin{tabular}{lclcc} 
& Lourdes & Intermédiaires & Légères & ref. \\
\hline - Chlamydomonas & 3 & $2(70-80)$ & 8 & $111(\mathrm{a})$ \\
- Paramecium & 3 & $3(65-65-81)$ & 8 & $107(\mathrm{a})$ \\
- Tetrahymena & 3 & $3(100-85-70)$ & 4 & 86 (a) \\
- Crassostrea gigas (Huitre) & 2 & $3(91-98-107)$ & n.d. & 106 \\
- Mytilus edulis (Moule) & 2 & $2(96-83)$ & 3 & 98 \\
- Spisula solidissima (Palourde) & 2 & $2(96-83)$ & 3 & 98 \\
- Oursin & 2 & $3(\mathbf{1 2 2 - 9 0 - 7 6 )}$ & 4 or 6 & $8 ; 69\left(^{*}\right)$ \\
- Truite (Salmo gairdnerii) & 2 & $5(85-73-65-63-57)$ & 6 & 26
\end{tabular}

Dynéines des bras externes depuis les protistes jusqu'aux vertébrés. Les chaînes lourdes ont un poids moléculaire supérieur à $400 \mathrm{kDa}$; le poids moléculaire des chaînes intermédiaires est indiqué entre parenthèse. n.d. non déterminé.

${ }^{*}$ ) chez l'oursin Tripneustes gracilla 4 chaînes légères sont reportées [8] alors que chez l'oursin Strongylocentrotus purpuratus il y en aurait 6 [69].

(a) Dynéines provenant de cils, les autres proviennent de flagelles de spermatozoïdes. Voir aussi ref. [114].

diaires, bien que pour ces dernières des relations immunologiques existent dans différentes espèces (voir plus loin).

Les particules de $20 \mathrm{~S}$ se dissocient lorsqu'elles sont dialysées contre un milieu à faible force ionique $[49,50,102]$. Après une nouvelle séparation par ultracentrifugation sur gradient de saccharose, des particules migrant à $11 \mathrm{~S}$ avec une activité ATPasique sont obtenues. Ces particules ou sous-unités contiennent chacune une chaîne de haut poids moléculaire associée, pour certaines, avec des chaînes intermédiaires et légères. Un complexe formé par des chaînes intermédiaires et légères migrant à environ 9S peut aussi être isolé, ce complexe n'a pas d'activité ATPasique [102].

\section{b) Les dynéines des bras internes.}

Les bras internes d'axonème d'oursin peuvent être extraits par traitement à faible force ionique, en présence d'ATP, à partir d'axonème extrait au préalable par haute force ionique [92, 114]. Au moins quatre chaînes de haut poids moléculaires sont ainsi obtenues. Un complexe migrant à $21 \mathrm{~S}$ composé par deux de ces chaînes, deux chaînes intermédiaires et au moins quatre chaînes légères peut être isolé [115].

Chez Chlamydomonas, les études biochimiques de l'axonème normal et de ceux provenant de mutants dont les bras externes ou internes sont absents, indiquent qu'au moins six (et peut être huit) chaînes lourdes différentes participent à la constitution des dynéines de bras internes $[45,81,83,84$, 92]. Le bras I1 contient une particule migrant à $21 \mathrm{~S}$, contenant deux chaînes lourdes et deux chaînes intermédiaires. Les bras I2 et I3 semblent aussi chacun contenir 2 chaînes lourdes mais celles-ci, après extraction, sédimentent toutes à $11 \mathrm{~S}$ [83, 95]. Les chaînes lourdes qui composent les dynéines des bras I2 et I3 ne sont pas les mêmes dans la partie proximale et la partie distale de l'axonème, par contre les chaînes lourdes contenues dans le bras I1 gardent une composition constante [84]. Les bras internes sont donc constitués d'au moins cinq particules de composition différente le long de l'axonème de Chlamydomonas. Chez cette espèce les dynéines des bras 
internes semblent associées avec une protéine de $42 \mathrm{kDa}$; cette protéine a été identifiée comme étant de l'actine [82].

\section{c) Les chaînes lourdes.}

Différentes approches expérimentales ont montré que l'activité ATPasique des dynéines réside dans les chaînes de hauts poids moléculaires. Une technique a été d'utiliser des dérivés d'ATP photoréactifs (a P32-2azido-ATP et a P32-8azido-ATP) afin de marquer radioactivement le site actif. Ces composés sont liés, après réaction, aux chaînes lourdes [49]. Une autre technique, abondamment utilisée, est la photolyse des chaînes lourdes qui se produit près du site actif sous l'effet des ultra-violets en présence d'ATP, de $\mathrm{Mg} 2+$ et de vanadate (qui est un inhibiteur des dynéine-ATPases) [49]. Dans ces conditions, un seul site de coupure est observé, et deux fragments de la chaîne lourde sont séparés sur gel d'électrophorèse dénaturant.

Récemment, des informations complémentaires ont été obtenues à partir de l'analyse des séquences nucléotidiques de certaines des chaînes protéiques de haut poids moléculaires $[3,31,67,73,109]$. La première à avoir été clonée et séquencée entièrement est la chaîne $\beta$ de dynéine des bras externes des cils de la blastula d'oursin. Depuis les séquences complètes des chaînes $\beta$ et $\gamma$ ainsi qu'une séquence partielle de la chaîne $\alpha$ ont été obtenues chez Chlamydomonas. Ces chaînes sont codées par des ARN messagers de plus de $13 \mathrm{~kb}$, soit une séquence protéique de plus de 4000 acides aminés. Une des plus surprenantes observation est que ces séquences contiennent quatre motifs du site de liaison à l'ATP. Un de ces sites semble particulièrement bien conservé. Il s'agit du premier de ces sites, ou site P1, codant pour le motif protéique GPAGTGKT qui est exactement le même dans toutes les chaînes lourdes de dynéines flagellaires mais aussi cytoplasmiques analysées [109]. Ce site est aussi situé très près de point de clivage en présence de vanadate et d'ATP. Ces résultats laissent supposer qu'il s'agit $\mathrm{du}$ seul site fonctionnel en tant qu'ATPase.
Un second site, le quatrième $(\mathrm{P} 4)$, n'est relativement conservé que parmi les chaînes flagellaires. Le rôle des sites P2, P3 et $\mathrm{P} 4$ reste à déterminer.

Chacune des chaînes lourdes est un isoforme spécifique codé par un seul gène. La recherche des messagers homologues à l'aide de sondes contenant la région du site P1 a montré que chez la blastula d'oursin 11 gènes différents codent pour les chaînes lourdes de dynéine ciliaires [32]. Chez Chlamydomonas, cinq ont été trouvés [67,109], et chez Paramécium au moins sept [32]. La comparaison des séquences montre qu'il y a une plus grande homologie entre la chaîne $\beta$ d'oursin et de la chaîne $\beta$ de Chlamydomonas qu'entre cette dernière et les chaînes $\alpha$ et $\gamma$ provenant du même bras externe [109]. Chez la Drosophile, une sonde du site P1 de la chaîne $\beta$ de blastula d'oursin reconnaît un gène au niveau de la région contrôlant la fertilité sur le chromosome Y [28].

Les chaînes protéiques de hauts poids moléculaires provenant des dynéines de bras internes semblent avoir des propriétés similaires à celles des bras externes. Leur activité ATPasique est cependant plus faible [26, 44, 115]. Pour l'instant aucune séquence complète n'a été publiée pour ces chaînes lourdes, mais certains des clones obtenus en utilisant la séquence du site ATPasique comme sonde, codent certainement pour des isoformes de ces chaînes [32, 109].

\section{d) Les châ̂nes intermédiaires (IC).}

Des protéines avec des masses comprises entre 50 et $150 \mathrm{kDa}$ sont associées aux particules de dynéines dérivées des bras internes et externes (Tableau 1). Des homologies immunologiques et au niveau des séquences protéiques ont été montrées entre les chaînes intermédiaires de bras externes de différentes espèces phyllogénétiquement éloignées (Figure 4). Un anticorps monoclonal dirigé contre la chaîne $\mathrm{IC} 2$ de $70 \mathrm{kDa}$ associé à la dynéine du bras externe de Chlamydomonas réagit avec une chaîne de $73 \mathrm{kDa}$ de dynéine du bras exter- 


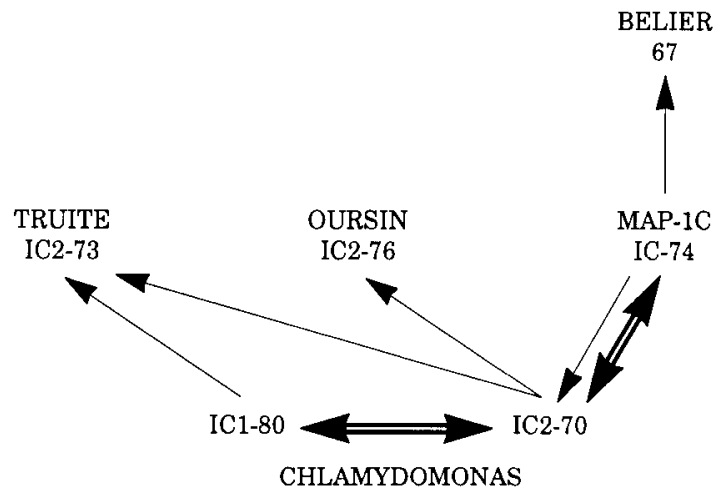

Figure 4 : Relation entre chaînes intermèdiaires. Les relations entre les différentes chaînes intermédiaires des dynéines de bras externes ou de dynéine cytoplasmique (MAP1C) sont indiquées (voir texte). Les simples flèches indiquent des croisements immunologiques, les fléches doubles des homologies de séquences.

ne de spermatozoïde de truite. Cet anticorps reconnaît aussi une chaîne intermédiaire de $76 \mathrm{kDa}$ chez la dynéine externe de spermatozoïde d'oursin [50]. La chaîne de $73 \mathrm{kDa}$ de spermatozoïde de truite est aussi reconnue par un autre anticorps monoclonal dirigé contre la chaîne IC1 (80 kDa) de Chlamydomonas [50]. De même, un anticorps monoclonal contre une chaîne intermédiaire de $74 \mathrm{kDa}$ de dynéine cytoplasmique réagit avec la chaîne de $70 \mathrm{kDa}$ chez Chlamydomonas mais aussi avec une chaîne de 67 $\mathrm{kDa}$ dans le flagelle de spermatozoïdes de bélier [77]. Au niveau des séquences nucléotidiques, des homologies bien que faibles existent entre les séquences des chaînes 70 et $80 \mathrm{kDa}$ de Chlamydomonas, mais aussi entre la chaîne de 70 kDa et la chaîne intermédiaire cytoplasmique de $74 \mathrm{kDa}$ [65-77]. Il existe donc un certain nombre d'épitopes et donc de séquences conservés parmi les chaînes intermédiaires et des réarrangements entre diverses parties de ces chaînes ont eu lieu au cours de l'évolution.

Ces similarités sont certainement liées aux rôles particuliers assurés par ces chaînes dans l'assemblage et la fonction des dynéines. Chez Chlamydomonas, la chaîne de $70 \mathrm{kDa}$ est située à la base du bouquet et chez les mutants où elle est absente l'assemblage du bras externe ne se fait pas [65]. In situ, la chaîne de $70 \mathrm{kDa}$ ainsi que la chaîne de $78 \mathrm{kDa}$ sont à proximité du microtubule et semblent participer à la liaison entre celui-ci et la dynéine [51]. La chaîne de $70 \mathrm{kDa}$ interviendrait aussi dans le contrôle de l'activité de la dynéine durant le battement flagellaire [66].

\section{e) Les chaînes légères.}

Ces chaînes de petits poids moléculaires sont en nombre variable suivant l'espèce et la provenance de la dynéine (Tableau 1). Elles n'ont pas de fonction connue pour l'instant. Chez Chlamydomonas, une chaine légère reste fortement liée avec chacune des chaînes lourdes $\alpha$ et $\beta$ au cours des différentes étapes de dissociation. Cependant, ceci n'est pas général chez les autres dynéines de bras externe. Une partie des chaînes légères semble situer à la base du bras de dynéine en association avec des chaînes intermédiaires $[51,64]$.

\section{Les fibres radiaires}

Dans les axonèmes de Chlamydomonas mutants où les fibres radiaires sont absentes on remarque la disparition de 17 protéines (nommées de RSP1 à RSP 17) en gel bidimensionnel [39]. Ces protéines ont des poids moléculaires de 34 à $124 \mathrm{kDa}$. Cinq de ces protéines ont pu être localisées au niveau de la tête de ces fibres (RSP 1, 4, 6, 9, 10) [20]. Parmi les autres, RSP3 est particulièrement importante pour la liaison entre la fibre et le microtubule : chez les mutants où seule cette protéine est absente la fibre ne s'assemble pas au niveau de l'axonème [21]. Inversement, la protéine RSP3 est capable de se lier seule aux microtubules de mutants déficients en fibres radiaires.

Les séquences des protéines RSP3, RSP4 et RSP6 ont été analysées [20, 110]. Des homologies existent entre RSP4 et RSP6 mais pas avec d'autres protéines connues. 


\section{Les ponts de nexines}

Il n'existe qu'une seule étude présentant l'isolement et la purification de ces ponts. Il semble qu'ils soient, chez les spermatozoïdes d'invertébrés marins, formés par une seule molécule dont le poids moléculaire est d'environ $160 \mathrm{kDa}$ [98].

\section{Le manchon central}

Chez Chlamydomonas, 23 protéines différentes de la tubuline sont présentes dans le manchon central. Parmi ces protéines, une tektine a été immunologiquement détectée [72]. Récemment, il a été montré que cinq protéines proches de la kinésine font parties du manchon central [9, 25, 42, 108]. Deux d'entre elles ont des poids moléculaires de $110 \mathrm{kDa}$ et se localisent respectivement sur un des microtubules et le long de l'axe entre les deux microtubules $[9,42]$. Les kinésines forment une famille de moteur moléculaire qui transportent des vésicules le long des microtubules en présence de nucléotide triphosphate [79]. Chez Tetrahymena il semble que de la dynéine migrant à $14 \mathrm{~S}$ soit présente dans ce manchon [59].

\section{Autres composants de l'axonème}

\section{a) Les tektines}

Ces protéines sont proches du groupe des filaments intermédiaires. Dans l'axonème de spermatozoïdes d'oursin, elles forment un ruban associé à chacun des doublets externes [72]. Ce ruban se situe à la jonction entre le microtubule $\mathrm{A}$ et $\mathrm{B}$ près de l'endroit où s'ancrent les fibres radiaires. Trois tektines ont été mises en évidence (tektine A, B et C). Elles ont des poids moléculaires de 57-59 kDa pour la $\mathrm{A}, 51-52$ pour la $\mathrm{B}$ et 46-47 pour la C. Ces tektines sont aussi présentes au niveau des centrioles, du corps basal et d'un certain nombre d'autres structures microtubulaires des cellules.

\section{b) La calmoduline}

La présence de calmoduline au niveau de l'axonème de nombreuses espèces a été montrée [76]. La calmoduline est une protéine de 16 à $17 \mathrm{kDa}$ capable de lier 4 molécules de $\mathrm{Ca} 2+$ par molécule. Le rapport entre la calmoduline et la tubuline semble constant de 1 molécule pour 60 molécules. La localisation de la calmoduline au niveau de l'axonème n'est pas connue. La calmoduline et le calcium agissent sur le type de battement flagellaire (symétrie et fréquence) soit directement soit indirectement via la cascade protéinekinase phosphorylation. D'autres protéines capables de lier le calcium sont aussi présentes au niveau de l'axonème et du corps basal [76].

\section{Les structures accessoires}

\section{a) Les fibres denses}

Chez les spermatozoïdes de mammifères, ces neuf structures périaxonèmales particulièrement imposantes peuvent être isolées et purifiées. Elles sont constituées de protéines proches des kératines et sont très riches en ponts disulfures. Chez le spermatozoïdes de rat six phospho-protéines de 13 à $87 \mathrm{kDa}$ ont pu être isolées à partir de ces fibres et la séquence nucléotidique d'une de ces protéines a récemment été obtenue [15, 105]. A partir de fibres denses de spermatozoïdes humains, deux protéines de 55 et $67 \mathrm{kDa}$ ont été isolées, ces protéines ne semblent pas être phosphorylées [36].

\section{b) La gaine fibreuse}

Chez le rat, la gaine fibreuse est composée d'au moins 15 protéines, dont une majeure à $80 \mathrm{kDa}[17,74]$. Cette protéine est une phospho-protéine dont la séquence a été analysée. Cette séquence contient un site de reconnaissance de la protéine-kinase $A$, et des expériences ont montré que la sousunité régulatrice de cette protéine-kinase pouvait s'y fixer [17]. Ces résultats sont en accord avec les observations précédentes immunolocalisant les protéines kinases cAMP-dépendantes au niveau de la gaine fibreuse $[54,55,112]$. 


\section{ROLE DANS LE MOUVEMENT}

Du fait de leur petite taille et de leur masse quasi nulle, les cellules doivent vaincre la viscosité du milieu qui les entoure pour pouvoir ce déplacer [11].

Le battement des cils et des flagelles résulte d'un phénomène actif, dû à l'attachement temporaire des dynéines des bras d'un doublet externe au microtubule $\mathrm{B}$ du doublet adjacent. Cet attachement se fait lors de la fixation d'ATP par les chaînes lourdes de dynéine. Lors de l'hydrolyse de l'ATP, il se produit un déplacement des bras de dynéines qui induisent une translation du doublet adjacent. Lors de la libération d'ADP, qui est le facteur limitant de cette réaction, le bras de dynéine se relâche pour retourner à sa position initiale. Ce mécanisme à été exploré enzymologiquement, ce qui a permis de montrer que chacune des chaînes lourdes hydrolyse une molécule d'ATP lors du cycle. Plus récemment, d'autres détails ont été obtenus grâce à la mise au point d'un essai in vitro. Dans cette technique, la dynéine isolée est adsorbée sur une lame de verre et sert à déplacer des microtubules en présence d'ATP (essai de "glidding"). C'est ainsi que l'on à confirmé que les dynéines flagellaires ne sont capables de déplacer les microtubules que de façon orientée vers leur extrémité + , ce qui correspond à la partie distale du flagelle $[68,69,103,104]$.

Au niveau de l'axonème, le glissement interdoublet est transformé en courbure à cause des résistances que lui opposent certaines des autres structures axonémales et à l'ancrage basal qui oriente le glissement. Quand la courbure de l'axonème atteint une certaine valeur, la région courbée se déplace le long de l'axonème. Pendant ce temps, une courbure se développe sur le côté opposé de l'axonème. Pour expliquer ce phénomène régulier et continu plusieurs mécanismes liés par des rétro-actions doivent exister. De nombreux modèles théoriques et mathématiques tenant compte à la fois de l'énergie, des résistances externes et internes, et de divers différents autres facteurs ont été développés pour décrire ce mécanisme [12, $19,37,57,93]$, mais aucun n'est suffisant pour expliquer les différentes situations expérimentales rencontrées.

A ce jour si le mécanisme de base (le glissement) est admis et démontré directement par l'observation [13], les autres contrôles restent mystérieux. On peut supposer que c'est dans la géométrie de l'axonème que réside une grande partie de ces contrôles, ce qui semble en partie démontré par les nombreux résultats obtenus à l'aide de Chlamy. domonas mutants.

\section{Les microtubules}

Des microtubules isolés provenant de la polymérisation in vitro de tubuline de cerveau montrent une grande flexibilité. Lors des essais de "glidding" lorsque des longs microtubules qui sont en train de se déplacer heurtent un obstacle, ils se courbent de façon importante. Ils sont capables, lorsque assez de force a été accumulée pour vaincre l'obstacle, de se détendre très rapidement pour se redresser et continuer leur déplacement. Les microtubules possèdent une grande élasticité et une forte résistance à la courbure qui sont certainement nécessaires au cours du battement flagellaire.

\section{Les dynéines des bras externes et internes}

\section{a) Les dynéines des bras externes}

Certains types d'infertilité chez l'homme sont associés à des spermatozoïdes dont le battement flagellaire se produit à une fréquence réduite. Cette diminution de fréquence est souvent liée à l'absence des bras externes [1, 43]. Cette observation à été aussi faite avec des mutants de Chlamydomonas dont les bras externes sont absents [47]. Une démonstration directe du rôle des dynéines des bras externes dans la fréquence flagellaire a été obtenue par Gibbons et Gibbons [29]. Ces auteurs ont démembrané des spermatozoïdes d'oursin, puis les ont 
traités à haute force ionique. Après cette extraction des bras externes, les spermatozoïdes ont été réactivés en présence d'ATP. Ils présentent alors une fréquence de battement inférieure de deux fois à celle des spermatozoïdes non-extraits [24, 29]. Les dynéines des bras externes ne sont donc pas nécessaires pour la génération et la propagation du battement, mais leur présence accroît la force déployée par l'axonème pour vaincre la viscosité du milieu [14].

Des résultats récents suggèrent aussi que les dynéines des bras externes pourraient exercer un contrôle régulateur sur l'activité de celles des bras interne [53].

Les différentes sous-unités et donc les différentes chaînes lourdes qui forment la dynéine des bras externes n'ont pas les mêmes propriétés biochimiques et enzymatiques $[27,50]$. Chez le spermatozoïde d'oursin, la sous-unité formée par la chaîne $\alpha$ et celle par la chaîne $\beta$ plus les chaînes intermédiaires $(\beta / \mathrm{IC})$ n'ont pas non plus les mêmes propriétés motrices. Au cours des essais de "glidding", la sous-unité $\alpha$ est capable de maintenir les microtubules mais pas de les transporter, alors que la sous-unité $\beta / \mathrm{IC}$ les transporte mais n'est pas capable de les lier en absence d'ATP [68, 69]. Des résultats similaires ont été obtenues avec les sousunités provenant des bras externes de Tetrahymena [103, 104].

Ces rôles différents pour les différentes chaînes lourdes d'un même bras externe sont aussi suggérés par les résultats obtenus avec certains mutants de Chlamydomonas. L'absence de la chaîne lourde $\alpha$ se traduit par une mobilité intermédiaire entre un Chlamydomonas normal et un mutant sans bras externes [89]. Par contre, chez un mutant dont la chaîne $\beta$ est tronquée (l'absence complète de chaîne $\beta$ ne permet pas l'assemblage du bras externe), et donc où le bras externe contient les chaînes lourdes $\alpha$ et $\gamma$ associées aux chaînes intermédiaires, la mobilité est la même qu'en l'absence complète des bras externes [90]. Ceci indique que la chaîne $\beta$ complète est nécessaire au bon fonctionnement de tout le bras externe.

Certaines des chaînes lourdes de dynéine de bras externes sont phosphorylés in vivo et peuvent être phosphorylées in vitro $[18,22$, $35,52,99,107]$. Chez les spermatozoïdes de Ciona, la phosphorylation de chaîne(s) lourde(s) semble corrélée à l'activation de la mobilité in vivo. Cependant, in vitro cette phosphorylation n'est pas suffisante pour activer la mobilité, d'autres facteurs cytoplasmiques sont nécessaires [22]. Chez Chlamydomonas, mais aussi chez les spermatozoïdes de mollusques, in vivo seule la chaîne $\alpha$ est phosphorylée [52, 99]. Par contre, dans les cils des branchies de mollusques et les cils de Tetrahymena se sont des chaînes de faible poids moléculaire associées au dynéines qui se phosphorylent [35, 99, 107]. La phosphorylation des chaînes de dynéine n'a pratiquement aucun effet sur l'activité ATPasique mais semble augmenter la vitesse de translocation des microtubules au cours des essais de "glidding" [35]. Chez Chlamydomonas et Paramecium, il existe une kinase endogène spécifique, qui co-migre avec la dynéine dans les gradients de saccharose.

\section{b) Les bras internes}

Puisque le battement flagellaire peut se produire en l'absence des bras externes, cela suppose que les bras internes sont suffisants pour produire un battement coordonné. L'étude de mutants de Chlamydomonas, dont certains types de bras internes sont spécifiquement absents, apportent des données complémentaires.

En l'absence des bras I1 ou I2, une diminution de l'amplitude de la courbure lors du battement se produit mais la fréquence de battement ne change pas $[47,48]$. Les bras I3 et externes sont donc suffisants pour produire un battement, et les bras 11 et I2 n'interviennent que dans la forme de ce battement.

In vitro, les dynéines isolées de bras externes sont capables de déplacer les 
microtubules durant les essais de "glidding". Certaines produisent une rotation des microtubules pendant leur mouvement [45]. Cette propriété semble vraie aussi pour les dynéines des bras internes de Tetrahyména [103, 104]. Le rôle de cette propriété dans le battement flagellaire est pour l'instant inconnu.

\section{Les ponts de nexine}

Les ponts de nexine maintiennent les doublets externes entre eux. Il a été montré que chez des axonèmes démembranés, traités par de la trypsine puis réactivés en présence d'ATP, les doublets externes deviennent capables de glisser librement les uns par rapport aux autres et sont extrudés de l'axonème [113]. Quand ces axonèmes sont examinés en microscopie électronique, les principales structures dégradées sont les ponts de nexine et les fibres radiaires. Ces deux strucutures semblaient donc responsables de la transformation du glissement en courbure. Récemment, l'obtention chez Chlamydomonas de mutants dont les fibres radiaires sont absentes mais qui présentent un battement flagellaire (cf. plus loin), a fait des ponts de nexine, le principal acteur de ce mécanisme [85, 87].

Des études en microscopie électronique avaient suggéré que les ponts de nexine pouvaient s'étirer au cours du glissement (jusqu'à plus de $100 \mathrm{~nm}$ ), mais des observations plus récentes contestent ces résultats. Il semble en fait que les ponts de nexine glisseraient le long du microtubule au cours du battement [10]. La façon précise dont les ponts de nexine agissent pour produire la courbure reste à déterminer.

\section{Les fibres radiaires}

Les mutants de Chlamydomonas, à qui manquent les fibres radiaires, sont immobiles; cependant, une mutation supplémentaire peut faire réapparaître le battement sans restaurer ces fibres. Ces mutations dites suppressives se produisent au niveau de protéines axonémales qui sont regrou- pées sous le nom de "complexe régulateur des dynéines $(\mathrm{crd}$ )" (dynein regulatory complex) $[85,87]$. Ce groupe de protèines semble intervenir dans la transmission des informations depuis les fibres radiaires vers les complexes de dynéines. Ceci est aussi montré par le fait que des dynéines internes, ayant été en contact avec des fibres radiaires, sont capables de transporter les microtubules durant les essais de glidding plus rapidement que celles provenant de mutant où les fibres sont absentes [96, 97]. Les fibres radiaires interviennent donc dans le battement, via l'action d'autres protéines, mais ne sont pas absolument nécessaires pour que celui-ci ait lieu.

\section{Le manchon central.}

Chez certains eucaryotes simples, le manchon central est beaucoup plus long que les doublets externes et est observable au bout du flagelle: ce manchon central tourne durant le battement flagellaire et joue un rôle direct dans la mobilité [75]. Cependant, ceci est un cas particulier; il semble en effet que le manchon central ne tourne pas dans la plupart des espèces. Inversement, certaines espèces présentent des axonèmes de type $9+0$, comme le spermatozoïde d'anguille. Ces spermatozoìdes ont un battement flagellaire tridimensionel avec une fréquence de $90 \mathrm{Hertz}$ [30]. La comparaison du battement flagellaire du spermatozoïde de Limule américaine, qui possèdent un axonème $9+2$, avec celui de Limule asiatique, à axonème $9+0$, indique aussi un rôle du manchon central dans la formation d'un battement plan [40].

Chez Chlamydomonas, les mutants sans paire centrale sont immobilisés. Cependant, comme pour ceux sans les fibres radiaires, une mutation supplémentaire au niveau du crd peut restaurer la mobilité sans réparer la structure axonèmale [96, 97]. Le manchon central n'est donc pas essentiel au battement mais il peut contrôler l'activité axonèmale, certainement via les fibres radiaires, et intervenir peut être dans la formation d'une vague bidimensionnelle. 


\section{Le "complexe régulateur des dynéines"}

La découverte de ce complexe de protéines axonémales provient de la suppression d'immobilité chez les mutants sans fibres radiaires ou sans doublet central qui se produit lors de la mutation d'une de ces protèines. La plupart de ces protéines si elles sont mutées seules ne produisent pas d'effet sur la mobilité ou produisent une mobilité similaire à celle de mutant sans bras interne I2 et I3. Actuellement, sept polypeptides de 29 à $129 \mathrm{kDa}$ forment une partie de ce complexe [85]. Il a été aussi montré que des mutations ponctuelles sur des chaînes lourdes de dynéines de bras internes et externes (en particulier $b$ ) produisent le même effet suppresseur qu'une mutation dans le $\operatorname{crd}[85,87,88]$.

Le rôle de ce complexe protéique serait donc de faire passer des informations depuis le doublet central et les fibres radiaires pour moduler ou inhiber l'activité des bras de dynéine. Au niveau ultrastructural ce $\mathrm{crd}$ a été localisé sur le microtubule $\mathrm{A}$ des doublets externes. Il se situe à proximité des bras I2 et I3, entre la fibre radiaire S2 et le bras externe (Figure 2) [60,45].

\section{Les structures accessoires}

Le rôle des fibres denses et de la gaine fibreuse dans la mobilité des spermatozoïdes a été mis en évidence, principalement, par l'étude de spermatozoïdes humains présentant des défauts de ces structures [94]. Quand les fibres denses n'ont pas leur longueur normale ou quand la gaine fibreuse présente des défauts dans la localisation des colonnes, l'amplitude du battement flagellaire et la vitesse de déplacement des spermatozoïdes sont diminuées. Cependant la fréquence du battement flagellaire reste la même. Lindemann et al., [56] ont montré que chez le spermatozoïde de rat il existe une unité fonctionnelle entre la paire centrale, les doublets 3 et 8 et les fibres (et colonnes) qui leur sont associés. Cet ensemble forme un axe perpendiculaire à l'axe du battement flagellaire et délimite deux parties fonction- nelles de l'axonème [57].

Les structures accessoires semblent avoir un rôle dans la transformation du glissement en courbure, certainement par un apport de résistance au niveau des pièces intermédiaires et principale du flagelle.

\section{CONCLUSION}

Au cours de ces dernières années, les études biochimiques et ultrastructurales des mutants de Chlamydomonas ont permis de situer, de caractériser et de définir le rôle de certaines des protéines axonémales dans le battement. Il est maintenant nécessaire de confirmer ces résultats chez d'autres espèces ne présentant pas les même caractéristiques évolutives (ex. phototactismes [38]). L'oursin, où la biochimie axonèmale du spermatozoïdes et sa mobilité sont bien étudiées, pourrait être un modèle intéressant. Les mammifères, où la structure flagellaire est plus complexe, à cause des fibres denses et de la gaine fibreuse, pourraient aussi devenir d'une grande utilité grâce à l'obtention de mutants (par sélection ou par transgénèse) chez la souris [62, 80]. Enfin, l'étude détaillée de la mobilité et de la biochimie axonèmale de spermatozoïdes humains présentant des défauts génétiques pourrait apporter des informations complémentaires $[1,5,23,42]$.

Dans cette revue le contrôle de la mobilité par des facteurs externes n'a pas été directement abordé : le rôle du calcium et de l'AMPc dans l'initiation du mouvement et sur la forme flagellaire semble maintenant démontré $[7,55,101,112]$.

La découverte qu'un certain nombre de protéines sont capables de contrôler ou d'inhiber le mouvement en fait des cibles potentielles de la régulation par les facteurs externes.

\section{REFERENCES}

1. Afzeluis B.A. : Int. Rev. Exp. Pathology 19 : 1-43, 1979. 
2. Afzeluis B,A., Bellon P.L., Lanzavecchia S. et Dallai R. : In : Comparative Spermatology 20 Years After: The Axoneme Studied by Computeraided analysis, B. Baccetti ed., Serono Symposia, vol. 75, Raven Press, New York. 315-319, 1991.

3. Asai D.J., Beckwith S.M., Kandl K.A., Keating H.H., TJandra H., ET Forney J.D. : J. Cell Sci. $107: 839-847$, (1994).

4. BACCETTI B, : In : Biology of Fertilization, Vol. 2 : Evolution of the Sperm Cell, C.B. Metz, and A. Monroy eds, Academic Press, New York. 1-58, (1985).

5. Baccetti B, Burrini A.G., Pallini V., et Renieri T. : J. Cell Biol. 88 : 102-107. 1980.

6. Barkalow K., Avolio J, Holwill M.E.J., Hamasaki T., ET SATIR P. (1994) : Cell. Motil. Cytoskel. 27 : 299-312.

7. BEDFORD J.M., ET Hoskins D.D. : In : Marshall's Physiology of Reproduction, vol. 2 : The Mammalian Spermatozoon, G.E. Lamming ed. Churchill Livingstone, London. 379-569, 1990.

8. Bell C.W., Fronk E., et Gibbons I.R. : J. Supramol. Struct. $11: 311-317,1979$.

9. Berstein M., Beech P.L., Gatz S.G., et Rosenbaum J.L. : J. Cell, Biol. 125 : 1113-1326, 1994.

10. Bozkurt H.H., et Woolley D.M. : Cell Motil. Cytoskel., 24 : 109-118. 1993.

11. Bray D.: Cell Movements. Garland Publishing, New York. 1992.

12. Brokaw C.J. : In: Cell Movement, Vol. 1 : Operation and Regulation of the Flagellar Oscillator, F.D. Warner, P. Satir, et I.R. Gibbons eds. Alan R. Liss, New York. 121-140, 1989a

13. BROKAW C.J. : Science 243:1593-1596, 1989b.

14. BroKaW C.J. : Cell. Motil. Cytoskel. 28 : 199-204, 1994.

15. Burfeind, P., et Hoyer-Fender, S. : Dev. Biol. 148 : 195-204. 1991.

16. BuRns R.G., ET SuRRidge C.D. : In : Microtubules: Tubulin : Conservation and Structure, J.S. Hyams, et C.W. Lloyd, eds. Wiley-Liss, New York. 3-32, 1994.

17. Carrera A., Gerton G.L., et Moss S.B. : Dev. Biol. 165 : 272-284, 1994.

18. Chilcote T.J., et Johnson K.A. : J. Biol. Chem. $265: 17257-17266,1990$.

19. Cosson, J : Biol. Cell, 76 : 319-327,1992.

20. Curry A.M., Williams B.D., et Rosembaum J.L. : Mol. Cell. Biol. 12 : 3967-3977, 1992.

21. Deiner D.R., ANG L.H., et Rosembaum J.L. : J. Cell. Biol., 123 : 183-190, 1993.
22. Dey C.S., et BrokaW C.J. : J. Cell. Sci., $100: 815-$ 824, 1991.

23. Feneux D., Serres C., et Jounnnet P. : Fertil. Steril. 44 : 508-511, 1985.

24. Fox L.A., ET SALE W.F. : J. Cell. Biol. 105:1781$1787,1987$.

25. Fox L.A., Sawin K.E., et Sale W.F.: J. Cell. Sci. 107:1545-1550, 1994.

26. Gatti J.-L., King S.M., Moss A.G., Et Witman G.B. : J. Biol. Chem. 264 : 11450-11457, 1989.

27. GatTI J.-L., KING S.M., ET Witman G.B. : In : Comparative Spermatology 20 Years After: The ATPases of Chlamydomonas Outer Arm Dynein Differ in Their pH and Cationic Requirements, B. Baccetti ed., Serono Symposia, vol 75, Raven Press, New York. 373-375, 1991.

28. Gepner J., et Hays T.S. : Proc. Natl. Acad. Sci. USA, $90: 11132-11136,1993$.

29. Gibbons B.H., Et GibBons I.R. : J. Cell Sci. 13 : 337$357,1973$.

30. Gibbons B.H., Gibbons I.R, Et Baccetti B. : J. Submicrosc. Cytol. $15: 15-20,1983$.

31. Gibbons B.H., Gibbons I.R, Mocz G., et Asai D.J. : Nature 352: 640-643, 1991.

32. Gibbons I.R., Asai D.J., Tang W-J.Y., ET Gibbons B.H. : Biol. Cell 76 : 303-309, 1992.

33. Goodenough U.W., et Heuser J.E. : In : Cell Movement, Vol. 1 : Structure of the Soluble and In-Situ Ciliary Dyneins Visualized by Quick-Freeze Deep-Etch microscopy, F.D. Warner, P. Satir, et I.R. Gibbons eds. Alan R. Liss, New York. 121-140, 1989.

34. Greer, K., et Rosenbaum, J.L. : In : Cell Movement, Vol. 2 : Post-translational Modifications of Tubulin, F.D. Warner, et J.R. MacIntoch eds. Alan R. Liss, New York. 47-66, 1989.

35. Hamasaki T., Barkalow K, Richmond J., et Satir P. : Proc. Natl. Acad. Sci. USA 88 : 7978-7922, 1991.

36. Henkel, R., Stalf, T., Mertens, N., Miska, W., et SchILl, W.-B. : Int. J. Androl. 17 : 68-73. 1994.

37. Holwill M.E. : In : Cell Movement, Vol. 1 : Biophysical Properties of the Sliding Filement Mechanism, F.D. Warner, P. Satir, et I.R. Gibbons eds. Alan R. Liss, New York. 61-75, 1989.

38. Horst C.J., ET WitMan G.B. : J. Cell. Biol. 120 : 733-741, 1993.

39. Huang B., Ramanis Z., Piperno G., et Luck D.J.L. : J. Cell. Biol. $88: 80-88,1981$.

40. Ishijima S., Sekiguchi K., et Hiramoto Y. : Cell. Motil. Cytoskel. 9 : 264-270, 1988. 
41. Johnson K.A., et WaLl J.S. : J. Cell. Biol. 96 : 669 $678,1983$.

42. Johnson K.A., HaAs M.A., et Rosenbaum J.L. : J. Cell. Sci. 107 : 1551-1556, 1994.

43. Jouannet P., Escalier D., Serres C. et David G. J. Submicrosc. Cytol. $15: 67-71,1983$.

44. Kagami O., et Kamiya R. : Eur. J. Biochem. 187 441-446, 1990.

45. Kagami O., et Kamiya R. : J. Cell. Sci. 103 : 653$664,1992$.

46. Kamiya, R., et Hasegawa, E. : Exp. Cell Res. 173 : 299-304, 1987.

47. Kamiya R., Kurimoto E., Sakakibara H., et OKagaKI T. : In : Cell Movement, Vol. 1: A Genetic Approach to the Function of Inner and Outer Arm Dyneins, F.D. Warner, P. Satir, et I.R. Gibbons eds. Alan R. Liss, New York. 209-218, 1989.

48. Kamiya R., Kurimoto E., et Mu'o E. : J. Cell. Biol. 112 : 441-447, 1991.

49. King S.M., ET WitMan G.B. : In : Cell Movement, Vol. 1 : Molecular Sructure of Chlamydomonas Outer Arm Dyneins, F.D. Warner, P. Satir, et I.R. Gibbons eds. Alan R. Liss, New York. 61-75, 1989.

50. King S.M., Gatti J.-L., Moss A.G., et Witman G.B. : Cell Motil. Cytoskel. 16 : 266-278, 1990.

51. King S.M., Wilkerson C.G., et Witman G.B : J. Biol. Chem. 266 : 8401-8407, 1990.

52. King S.M. eT Witman G.B : J. Biol. Chem. 269 : 5452-5457, 1994.

53. Kurimoto E., et Kamiya R. : Cell Motil. Cytoskel. $19: 275-281,1991$.

54. Lieberman S.J., Wasco W., Macleod J., Paupard M.C. ET ORk G.A. : J. Cell. Biol. $107:$ 1809-1816, 1988.

55. Lindemann C.B., et Kanous K.S. : Arch. Androl. $23: 1-22,1990$.

56. Lindemann C.B., Orlando A., et Kanous K.S. : J. Cell Sci. 102 : 249-260, 1992.

57. Lindemann C.B. : Cell Motil. Cytoskel. 29 : 141154,1994

58. Luck, D.J.L. : J. Cell. Biol., 98 : 789-794, 1984.

59. MARCiese-RAgONA S.P. ET JohnSON K.A. : Electron Microsc. Rev. 1 : 141-153, 1988.

60. Mastronarde D.N., O'Toole E.T., McDonald K.L., McIntoch J.R. et Porter M.E. : J. Cell. Biol. 118 : 1145-1162, 1992.

61. Mendel.kow E., et Mendelkow E.M. : Curr. Opin. Struct. Biol. $4: 171-179,1994$.

62. Merlino G.T., Stahle C., Linton R., Mahon K.A. et Willingham M.C. : Genes \& Dev. 5 : 1395-1406, 1991.
63. Mitchell D.R et Rosenbaum J.L. : J. Cell. Biol. $100: 1228-1234,1985$.

64. Mitchell D.R et Rosenbaum J.L. : Cell Motil. Cytoskel. $6: 510-520,1986$.

65. Mitchell D.R. ET Kang Y. : J. Cell. Biol. 113 : 835$842,1991$.

66. MitChell D.R. et Kang Y. : J. Cell. Sci. $105: 1069$ 1078, 1993.

67. Mitchell D.R., et Brown K.S. : J. Cell. Sci., 107 : 635-644, 1994.

68. Moss A.G., GatTi J.-L., et Witman G.B. : J. Cell. Biol. 118: 1177-1188, 1992 (a).

69. Moss A.G., Sale W.S., Fox L.A. et Witman G.B. : J. Cell. Biol. 118 : 1189-1200, 1992 (b).

70. Multigner L., Gagnon J., Van Dorsselaer A. et Jов D. : Nature $360: 33-39,1992$.

71. Muto E., Kamiya R. et Tsukita S. : J. Cell Sci., 99 : 57-66, 1991.

72. NoRRANDER J.M., ET LinK R.W. : In : Microtubules: Tektins, J.S. Hyams et Lloyd C.W. eds. Wiley-Liss, New York. p 201-220 1994

73. OGAWA K. : Nature, 352 : 643-645. 1991.

74. OLSON G.E. : In : The Spermatozoon : Isolation of the Fibrous Sheath and Perforatorium of Rat Spermatozoa, D.W. Fawcett et J.M. Bedford eds, Urban-Schwarzenberg, Baltimore. p 395-400, 1979

75. Омото C.K. Ет Wiтman G.B. : Nature $288: 708-710$ 1981.

76. Otrer T. : In: Cell Movement, Vol. 1 : Calmodulin and the Control of Flagellar Movement, F.D. Warner, P. Satir, et I.R. Gibbons eds. Alan R. Liss, New York. p 281-298, 1989

77. Paschal B.M., Mikami A., Pfister K.K., et Vallee R.B. : J. Cell. Biol. 118 : 1133-1143, 1992.

78. Paturle-Lafanechere L., Manier M., Trigault N., Pirollet F., Mazargull H. et Job D : J. Cell Sci. $107:$ 1529-1543, 1994.

79. Peirera A. eT Goldstein L.S.B. : In: Microtubules : The Kinesin Superfamily, J.S. Hyams et Lloyd C.W. eds. Wiley-Liss, New York. p 229-249, 1994.

80. Pilder S.H., Olds-Clarke P., Phillips D.M. et SilVER L.M : Dev. Biol. 159 : 631-642, 1993.

81. Piperno G. : Cell Motil. Cytoskel. 17 : 147-149, 1990.

82. Piperno G., ET LuCK, D.J.L. : J. Biol. Chem., 254 : 2187-219, 1990.

83. Piperno G., Ramanis, Z., Smith, E.F., et Sale, W.S.: J. Cell. Biol. 110 : 379-389, 1990.

84. Piperno G. et Ramanis Z. : J. Cell. Biol. 112 : 701709, 1991. 
85. Piperno G., Mead C., LeDizet M. et Moscatelli A.: J. Cell. Biol. 125 : 1109-1117, 1994.

86. PorTer M.E. ET Johnson K.A. : J. Biol. Chem. 258 : 6575-658, 1983.

87. Porter M.E., Power J. et Dutcher S.K. : J. Cell. Biol. 118 : 1163-1176, 1992.

88. Porter, M.E., Knott, J.A., Gardner, L.C., MitCHELl, D.R., ET DUTCheR S.K .: J. Cell. Biol. 126, 1495-1507, 1994.

89. Sakakibara H., Mitchell D.R. et Kamiya R. : J. Cell. Biol. 113 : 615-622, 1991.

90. Sakakibara H., Takada S., KING S.M., WitMan G.B. ET KamiYa R. : J. Cell. Biol. 122 : 653-661 1993.

91. SAle W.S. : J. Cell. Biol. 102 : 2042-2052, 1986.

92. Sale W.S., Fox L.A., ET Milgram S.L. : In : Cell Movement, Vol. 1 : Composition and Organization of the Inner Row Dynein Arms, F.D. Warner, P. Satir, et I.R. Gibbons eds. Alan R. Liss, New York. p 89-102, 1989.

93. Satir P. : Comp. Biochem. Physiol. 94A : 351-357, 1989.

94. Serres C, Feneux D. et Jounnnet P. : Cell Motil. Cytoskel. $6: 68-76,1986$.

95. Smith, E.F., et Sale, W.S. (1991) : Cell Motil Cytoskel., $18: 258-268$.

96. Smith E.F. eт SALE W.S : Science 257:1557-1559, 1992.

97. Smith E.F. et Sale W.S : In : Microtubules Mechanisms of Flagellar Movement, J.S. HYAMS et C.W. LLOYD eds, WILEY-LISS, New York. p 381-392, 1994.

98. STEPHENS R.E.: In: Molecules and Cell Movement: Structural Chemistry of the Axoneme, S. INOUE et R.E. STEPHENS eds, RAVEN PRESS, New York. p 181-206, 1975.

99. Stephens R.E. et Prior G. : J. Cell. Sci. 103 : 9991012, 1992.

100. Tadaka S. ET KamiYa, R. : J. Cell. Biol., 126 : $737-$ 745, 1994.

101. Tamm S.L. et Terasaki M. : J. Cell. Biol. 125 : 1127-1135, 1994.

102. Tang W.-J.Y., Bell C.W., Sale W.S. et Gibbons I.R. : J. Biol. Chem. 262 : 17728-17734, 1982.

103. Vale R. et Toyoshima Y.Y. : Cell 52 : 459-469, 1988.

104. Vale R. eT Toyoshima Y.Y. : J. Cell Biol. 108 : 2327-2334, 1989.

105. Vera J.C., Brito M., Zuvic T. et Burzio L.O. : J. Biol. Chem. 259 : 5970-5977, 1984.

106. Wada S., OKuno M., Nakamura K.-I. ET Mohri H. : Biol. Cell 76, 311-317, 1992.

107. WalczaK C.E. et Nelson D.L. : J. Cell Sci. 106 : 1369-1376, 1993.

108. Walther Z., Vashishtha M. et Hall J.L. : J. Cell. Biol. 126 : 175-188, 1994.

109. Wilkerson C.G., King S.M. et Witman G.B. : J. Cell Sci. 107 : 497-506, 1994.
110. Williams B.D., Curry A.M. et Rosembaum J.L. : J. Cell. Biol. 109 : 235-245, 1989.

111. WITMan G.B. : In : Cell Movement, Vol. 1 : Composition and Molecular Organization of the Dyneins, F.D. Warner, P. Satir, et I.R. Gibbons eds. Alan R. Liss, New York. p 25-35, 1989.

112. WitMan G.B. : In : Ciliary and Flagellar Membranes: Introduction to Cilia and Flagella, R.A. BLOODGOOD ed. PLENUM PRESS, New York. $p$ $1-30,1990$.

113. Witman G.B., Plummer J. et Sander, G. : J. Cell. Biol. $76: 729-747,1978$

114. Witman G.B., Wilkerson C.G., ET King S.M : In : Microtubules: The Biochemistry, Genetics, and Molecular Biology of Flagellar dynein, J.S. HYAMS et C.W. LLOYD eds, WILEY-LISS, New York. Wiley-Liss, New York. p 229-249, 1994.

115. Yokota E. et Mabuchi I. : J. Cell Sci. 107 : 345 $351,1994$.

\section{ABSTRACT}

\section{Molecular Basis of axonemal Movement}

\section{GATTI J.-L., DAcheUX J.-L.}

Because of their small size, cells encounter fundamentally different physical constraints when they want to move in their surrounding media than when aquatic animals want to move in water. For cells, external viscosity is the main resistance while inertia plays almost no role; then in order to move, cells will have to produce continually a force against the viscous media. During the evolution, eukaryotic cells have gained specialised structures to efficiently propel them : cilia and flagella. These thread-like appendages produce repetitive beating which consists in propagation of waves from the bottom to the tip of these structures. Cilia generally show an asymmetrical beating while flagella have a more symmetrical bend propagation. Cilia and flagella contain an almost identical internal complex machinery : the axoneme. As a consequence, the mechanisms involved in the generation of the movement are identical although the precise regulation may be different.

In this paper, the reader will find a review of the molecular organisation of the ciliary and flagellar axoneme and of the role played by some of the constituting elements on the generation of the movement.

Key words : axoneme, dynein, cilia, flagella, spermatozoa, movement. 